\title{
Extension induced phase separation and crystallization in semidilute solutions of ultra high molecular weight polyethylene
}

Wingstrand, Sara Lindeblad; Imperiali, Luna; Stepanyan, Roman; Hassager, Ole

Published in:

Polymer

Link to article, DOI:

10.1016/j.polymer.2017.12.042

Publication date:

2018

Document Version

Peer reviewed version

Link back to DTU Orbit

Citation (APA):

Wingstrand, S. L., Imperiali, L., Stepanyan, R., \& Hassager, O. (2018). Extension induced phase separation and crystallization in semidilute solutions of ultra high molecular weight polyethylene. Polymer, 136, 215-223. https://doi.org/10.1016/j.polymer.2017.12.042

\section{General rights}

Copyright and moral rights for the publications made accessible in the public portal are retained by the authors and/or other copyright owners and it is a condition of accessing publications that users recognise and abide by the legal requirements associated with these rights.

- Users may download and print one copy of any publication from the public portal for the purpose of private study or research.

- You may not further distribute the material or use it for any profit-making activity or commercial gain

- You may freely distribute the URL identifying the publication in the public portal 


\section{Accepted Manuscript}

trit

\section{polymer}

Extension induced phase separation and crystallization in semidilute solutions of ultra high molecular weight polyethylene

Sara Lindeblad Wingstrand, Luna Imperiali, Roman Stepanyan, Ole Hassager

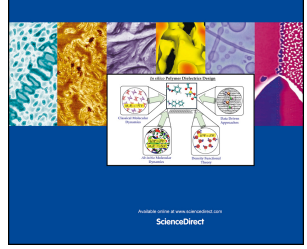

PII:

S0032-3861(17)31215-6

DOI:

10.1016/j.polymer.2017.12.042

Reference: JPOL 20231

To appear in: Polymer

Received Date: 8 September 2017

Revised Date: 7 December 2017

Accepted Date: 17 December 2017

Please cite this article as: Wingstrand SL, Imperiali L, Stepanyan R, Hassager O, Extension induced phase separation and crystallization in semidilute solutions of ultra high molecular weight polyethylene, Polymer (2018), doi: 10.1016/j.polymer.2017.12.042.

This is a PDF file of an unedited manuscript that has been accepted for publication. As a service to our customers we are providing this early version of the manuscript. The manuscript will undergo copyediting, typesetting, and review of the resulting proof before it is published in its final form. Please note that during the production process errors may be discovered which could affect the content, and all legal disclaimers that apply to the journal pertain. 


\title{
Extension Induced Phase Separation and
}

\section{Crystallization in Semidilute Solutions of Ultra High Molecular Weight Polyethylene}

Sara Lindeblad Wingstrand, ${ }^{\dagger}$ Luna Imperiali, ${ }^{\ddagger}$ Roman Stepanyan,$^{\ddagger}$ and Ole Hassager ${ }^{*, \dagger}$

$\dagger$ Technical University of Denmark, Department of Chemical and Biochemcial Engineering, Danish Polymer Center,DK-2800 Kgs. Lyngby, Denmark

$\ddagger D S M$ Research Performance Materials, Materials Science Center, NL-6160BB Geleen, Netherlands

E-mail: oh@kt.dtu.dk

\begin{abstract}
We investigate the influence of controlled uniaxial extension on various flow induced phenomena in semidilute solutions of ultra high molecular weight polyethylene (UHMwPE). Concentrations range from $9 \mathrm{w} \%$ to $29 \mathrm{w} \%$ and the choice of solvent is paraffin oil (PO). The start-up extensional behavior is measured at various Hencky strain rates $\dot{\varepsilon}$ and at two different temperatures $\left(150^{\circ} \mathrm{C}\right.$ and $\left.170^{\circ} \mathrm{C}\right)$ well above the melting point. At $\varepsilon>0.9$ the qualitative behavior of the samples differ significantly depending on the imposed conditions and the concentration of the samples. Overall we propose two flow scenarios: Scenario 1 - flow induced phase separation resulting in an unstable bulky filament and Scenario 2 - flow induced phase separation and crystallization resulting in a stable deformation and a smooth strongly strain hardening filament. Scenario 2
\end{abstract}


is observed only at $150^{\circ} \mathrm{C}$ at high $\dot{\varepsilon}$ and high concentrations. Scenario 1, observed at both temperatures, is most pronounced at low rates and/or high concentrations.

\section{Introduction}

Ultra high modulus polyethylene fibers are spun from solutions of ultra high molecular weight polyethylene (UHMwPE). ${ }^{1}$ To a large extent fiber spinning processes comprise uniaxial extensional upon extrusion and drawing of the polymeric liquid. The processability of UHMwPE solutions is thus determined by the rheological characteristics primarily in extension, yet controlled rheological studies of PE solutions are performed only in shear flow. ${ }^{2}$

In shear, UHMwPE solutions exhibit significant nonlinear characteristics. ${ }^{3}$ The long chains are easily deformed by flow causing the solutions to be highly shear thinning. Several studies on UHMwPE solutions utilize paraffin oil (PO) as solvent. It is a convenient solvent due to its low volatility and the fact that the chemical composition is the same as that of UHMwPE. Extensive work on UHMwPE/PO solutions in shear have been performed by Murase and co-workers. ${ }^{4-7}$ Apart from significant shear thinning they discovered other highly nonlinear phenomena in these systems. They found that multiple states of heterogeneities can be initiated by flow under the right conditions. At high shear rates the UHMwPE/PO solutions experience concentration fluctuations that eventually develop into actual phase separation. ${ }^{4}$ In addition, at temperatures close to the melting point $T_{m}$, the UHMwPE rich phase crystallizes into highly oriented structures. ${ }^{5,6}$ These flow induced phenomena have been found to play a huge role in the structural development of UHMwPE fibers during processing $^{7}$ as well as the final fiber strength. ${ }^{8,9}$ Unfortunately, the deformation in a spin-line is ill defined and lacks control of the deformation. As a result, the imposed deformation is quantified in terms of take-up speed or other measures related to the instrument rather than local deformation of the material. Due to experimental challenges regarding both control of the deformation and the non-stick nature of the sample, studies on polyethylene solutions in 
controlled extensional flows have, to our knowledge, never been performed.

The purpose of the present study is to characterize solutions of UHMwPE in controlled uniaxial extension. The solvent is PO and the samples are measured at constant deformation rates at temperatures well above $T_{m}$. Extension of these non-sticky samples are performed using a filament stretch rheometer (FSR) with a modified sample plate design to prevent slip off. Conditions under which flow induced phase separation and flow induced crystallization (FIC) occur are identified using simultaneous high-speed imaging. We map the regimes under which the different flow induced phenomena occur with respect to imposed deformation rate, polymer concentration, and temperature.

\section{Materials and method}

UHMwPE with $M_{w}=3500000 \mathrm{~g} / \mathrm{mol}$ and a broad molar mass distribution, supplied by DSM and paraffin oil (containing $0.2 \mathrm{w} \%$ antioxidant: 2,6-di-tert-butyl-p-cresol) were mixed in an extruder at a temperature well above the melting temperature. Three solutions were prepared containing $5 \mathrm{w} \%, 10 \mathrm{w} \%$ and $20 \mathrm{w} \%$ UHMwPE. The solutions were extruded directly into a mould and moulded into discs of diameter $D_{0}=8 \mathrm{~mm}$ and height $h_{0}=6 \mathrm{~mm}$.

\section{Thermogravimetric Analysis (TGA) and Differential Scanning Calorimetry (DSC)} Upon cooling to room temperature, some solvent was expelled from the sample due to crystallization of the UHMwPE. Hence, before starting rheological characterizations, the concentration of polymer in the remaining gel samples was determined using TGA (Discovery TGA from TA Instruments). Concentrations were determined from the integrals under the clearly separated PO and UHMwPE peaks (see Supplemental Material for details). The melting point was determined by DSC with an auto sampler from TA instruments (Discovery DSC from TA instruments). In Table 1 specifications regarding polymer concentration obtained from TGA and nominal melting temperature $T_{m}$ obtained from DSC are given. Table 1 also presents the average number of entanglements $Z$ and the Flory-Huggins predicted equilib- 
rium melting point $T_{m}$. The number of entanglements is given by $Z=\left(M_{w} / M_{e}\right) \phi^{\alpha}$ where the molar mass between entanglements $M_{e}=1120 \mathrm{~g} / \mathrm{mol}^{10}$ (Note here we assume a dilution exponent $\alpha=1) . T_{m}$ is given by ${ }^{11}$

$$
\frac{1}{T_{m}}-\frac{1}{T_{m}^{0}} \cong \frac{R}{\Delta H_{f}}\left[(1-\phi)-\chi\left(1-\phi^{2}\right)\right]
$$

The equilibrium melting point of the bulk polymer $T_{m}^{0}$ is set to $145^{\circ} \mathrm{C}$ and $\Delta H_{f}=3900 \mathrm{~kJ} / \mathrm{mol}$ is the heat of fusion. ${ }^{5}$ The interaction parameter $\chi$ is set to 0 as the chemical composition of $\mathrm{PE}$ and $\mathrm{PO}$ is the same.

Table 1: Material characteristics of the prepared solutions of UHMwPE in paraffin oil.

\begin{tabular}{l|c|c|c|c}
\hline \hline Sample & $\phi[w \%]$ & $T_{m}^{a)}\left[{ }^{\circ} \mathrm{C}\right]$ & $T_{m}^{b)}\left[{ }^{\circ} \mathrm{C}\right]$ & $Z$ \\
\hline $\mathrm{PE} / \mathrm{PO}-29 \%$ & 28.7 & 123.5 & 119.2 & 960 \\
\hline $\mathrm{PE} / \mathrm{PO}-17 \%$ & 16.8 & 121.6 & 115.8 & 560 \\
\hline $\mathrm{PE} / \mathrm{PO}-9 \%$ & 8.8 & 118.9 & 113.6 & 293 \\
\hline \multicolumn{5}{c}{ from DSC } \\
$\begin{array}{l}\text { b) } \\
\text { from Eq. (1) }\end{array}$
\end{tabular}

Melting temperatures measured by DSC are higher than predicted by Eq. 1. This difference is expected as melting of the PE crystals during the DSC heating ramp does not take place instantaneously. Kinetic delay pushes the melting to higher temperatures in the DSC and thus it tends to overestimate the $T_{m}$.

Small Angle Oscillatory Shear (SAOS) The linear rheology of the solutions was measured in SAOS. An ARES G2 rheometer from TA Instruments was used with a $25 \mathrm{~mm}$ plate-plate geometry. To prevent degradation of the sample, measurements where carried out in the presence of nitrogen. The samples were heated to $170{ }^{\circ} \mathrm{C}$ and left to relax. Frequency spectra was collected for $T=150{ }^{\circ} \mathrm{C}$ and $170^{\circ} \mathrm{C}$. At higher temperatures the loss of solvent was so significant that the rheology no longer was reproducible. Frequency sweeps obtained at different temperatures were shifted and combined into one master curve for each sample. 


\section{Combined Filament Stretch Rheometry and High-Speed Imaging of non-sticky samples}

Extensional rheometry was carried out on a VADER 1000 filament stretch rheometer (FSR) from Rheofilament. The advantage of the FSR is that is allows for controlled deformation to large deformations $\varepsilon>7$ via a feed back loop. ${ }^{12}$

The FSR comprises a moving top plate and a stationary bottom plate between which the sample is sandwiched. Upon movement of the top plate, the fluid is extended uniaxially due to the no slip boundary condition on the plates. ${ }^{13}$ The deformation is measured in the mid-filament plane using a laser micrometer and the response of the fluid is monitored via a force cell mounted on the bottom plate. The measure for deformation and response of the fluid is Hencky strain $\varepsilon$ and first normal stress difference $\sigma_{z z}-\sigma_{r r}$, respectively:

$$
\begin{gathered}
\varepsilon=-2 \ln \frac{D(t)}{D_{0}} \\
\sigma_{z z}-\sigma_{r r}=\frac{F(t)-\frac{1}{2} m_{f} g}{\frac{\pi}{4} D(t)^{2}}
\end{gathered}
$$

Here $D(t)$ and $D_{0}$ are the measured and initial diameter, respectively. $F(t)$ is the force on the bottom plate, $m_{f}$ is the mass of the filament and $g$ is the gravitational acceleration. Eq. (3) does not account for the shearing contribution due to the no-slip boundary condition on the plates. ${ }^{14}$ The reason for omitting this contribution is that we did not use standard flat plates for this study as discussed in the following section.

Plate design for measuring non-sticky samples The FSR-technique relies on the sample sticking to the end plates during the stretch experiment. Slip-off is an issue even for the most studied polymer in the FSR, polystyrene (PS). With PS, it is possible to circumvent this problem by increasing the prestretch i.e. increasing the area that sticks to the plate relative to the area of the midfilament plane. Increasing the prestretch was not sufficient in the case of the $\mathrm{PE} / \mathrm{PO}$ solutions as the presence of PO resulted in greasy samples 
that slipped off very easily. To overcome slip-off, several new plate geometries were designed. The best design is shown in Figure 1a and is in the following referred to as the "mushroom plate". The mushroom plate differs from the standard plate (Figure $3 \mathrm{~b}$ ) in the way that it has an extra plate on top of the standard plate separated by a small rod. The additional plate and rod is referred to as the "mushroom". The mushroom plate required a special
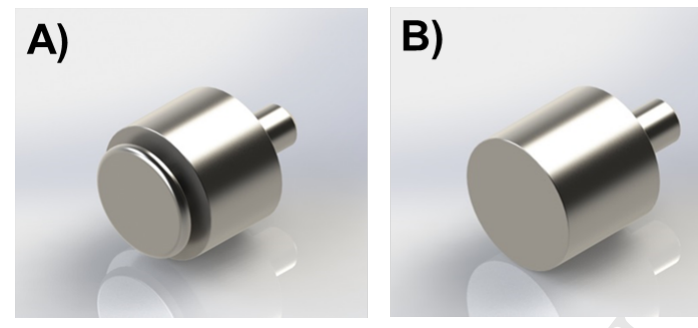

Figure 1: Sketch of plate designs. (a) The mushroom plate design developed for stretching of non-sticky samples. (b) The original plate design for sticky samples.

moulding procedure inside the FSR in order to anchor the "mushroom" into the fluid. The moulding procedure is illustrated in Figure 2. First the sample disc was sandwiched between the two plates and thick tin foil was wrapped around the plates. Second, the sample was heated to $165^{\circ} \mathrm{C}$ and the top plate was lowered so that the polymer filled the entire space. As a result, the sample encapsulated the mushroom such that it acted as an anchor upon stretching.

\section{Filament Stretching at a Constant Strain Rate with Simultaneous High-Speed}

Imaging We conducted experiments at a constant Hencky strain rate $\dot{\varepsilon}$ such that throughout the entire experiment the strain increased linearly with time: $\varepsilon=\dot{\varepsilon}$. The procedure for each stretch experiment was as follows. After moulding in the FSR as described in the previous section, the sample was heated to $165^{\circ} \mathrm{C}$ where it was pre-stretched and relaxed for $15-60$ min depending on the relaxation times of the sample. The temperature was then changed to the experimental temperature at which the sample was stretched at a constant rate. High-speed imaging of the sample during stretch was performed with a high-speed camera (FASTCAM Mini UX100 from Photron) and a LED light source. Both where placed 


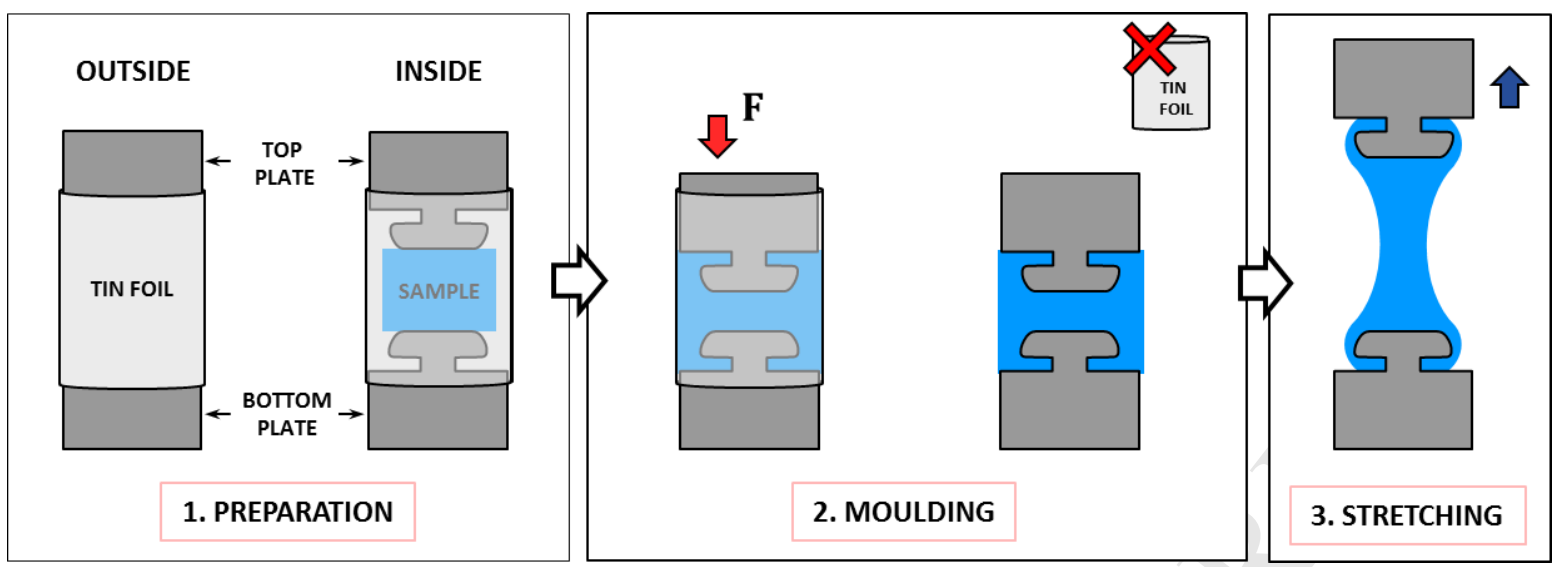

Figure 2: Sketch of moulding procedure in the FSR using the mushroom plates. 1. Preparation: Initial placement of the polymer disc between the bottom and top plates with a piece of tin foil wrapped around it. 2: Moulding: (left) Moulding procedure where the sample is heated above $T_{m}$ and the top plate is lowered by applying a force $\mathrm{F}$. (right) after moulding where the tin foil has been removed. 3. Stretching: The anchoring effect of the mushroom during stretching preventing slip-off.

on the same side of the sample where windows in the oven enabled the stretch to be captured. The frame rate was relatively low (50-1000 fps) and determined by the stretch rate of the given experiment - higher strain rates required higher frame rates.

\section{Results and Discussion}

\section{Linear Rheology}

The linear rheology of the three samples along with respective multimode Maxwell model fits are seen in Figure 3a. The response is given in terms of storage and loss moduli $G^{\prime}$ and $G^{\prime \prime}$, respectively. The first crossover is captured for $\mathrm{PE} / \mathrm{PO}-9$ while for $\mathrm{PE} / \mathrm{PO}-17$ and $\mathrm{PE} / \mathrm{PO}-$ 29 the first crossover are estimated by extrapolating G', G" to lower frequencies. The inverse value of the estimated first crossover frequency gives an indication of the average relaxation time by reptation $\tau_{d}$. Due to instrument limitations it was not possible to reach the terminal regime of the samples (the region where G' and G" reach a slope of 2 and 1, respectively) or the second crossover found at higher frequencies. For the three samples the average reptation 
time is in the order of $10^{2}-10^{3} \mathrm{~s}$.

The vertical shift between the samples arise from differences in concentration. That is in accordance with the tube model stating that the plateau modulus $G_{N}^{0}$ scales with $\phi$ as follows $G_{N}^{0}(\phi) \propto \phi^{1+\alpha}$ with the dilution exponent $\alpha=1$ or $1.3 .{ }^{15,16}$ The plateau modulus is the value observed in $G^{\prime}$ for a monodisperse well entangled polymeric liquid. For undiluted polymers this value is a material constant and for $\mathrm{PE}$ at $150^{\circ} \mathrm{C}$ the value is $G_{N}^{0}=2.6 \mathrm{GPa} \cdot{ }^{17}$ The plateau region is not clearly identified in these samples both due to polydispersity and due to the limited range of experimentally accessible frequencies $\omega$. As an approximation for $G_{N}^{0}$ we use $G_{\max }^{\prime}$ which is the value of $G^{\prime}$ at the highest measured frequency where the smallest slope of $G^{\prime}$ for all samples is measured. Figure $3 \mathrm{~b}$ compares the value of $G_{N}^{0}$ predicted by the tube model with the approximated values using $G_{m a x}^{\prime}$. The approximated values are relatively close to the prediction. For the given concentration range the value of $\alpha$ is expected to be 1 . In that case, the approximated values increasingly diverge from the prediction as the concentration decrease. That is expected since $G^{\prime}$ at lower concentrations show a less pronounced plateau compared to the higher concentrations.

According to the tube model the reptation time of a highly entangled linear polymer melt is given by $\tau_{d}=\tau_{e} Z^{3}$ where $\tau_{e}$ is the relaxation time of one entanglement segment. ${ }^{18}$ Moreover, dilution increases the distance between entanglements along the chain, thus increasing $\tau_{e}$. If the solvent and the polymer are isofrictional, the entanglement relaxation time of a diluted polymer chain scales with $\phi^{-2 \alpha}$. Since $Z \propto \phi^{\alpha}$, the scaling of the reptation time with concentration is $\tau_{d} \propto \phi^{\alpha}$. Figure 3c shows the evolution of $\tau_{d}$ estimated from the first crossover with concentration. Evidently $\tau_{d}$ appears to follow the prediction by the tube model quite nicely using $\alpha=1$. The fact that the systems obey the tube model suggests that $\mathrm{PE}$ and $\mathrm{PO}$ are isofrictional at equilibrium which is expected since the repeat units of $\mathrm{PE}$ and $\mathrm{PO}$ are identical.

Figure 4 shows the normalized linear response of the three solutions. The normalization is performed in accordance with the tube model. Vertically, the data is normalized by 

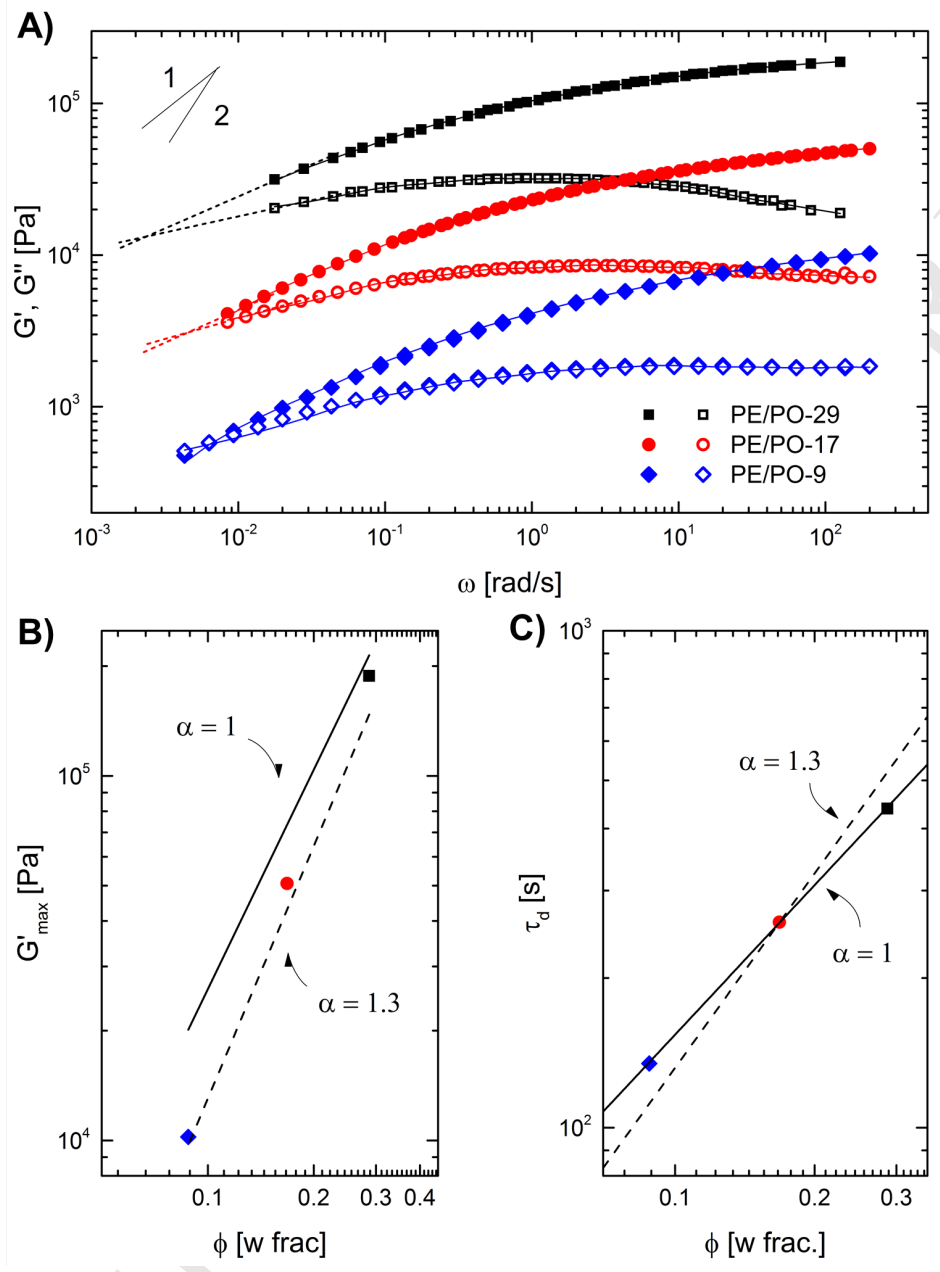

(a)

Figure 3: Linear response of $\mathrm{PE} / \mathrm{PO}$ solutions at $150^{\circ} \mathrm{C}$. (a) Dynamic frequency sweep expressed in terms of storage modulus $G^{\prime}$ (closed symbols) and loss modulus $G^{\prime \prime}$ (open symbols). Solid lines represent best fit of the multi mode Maxwell model, dashed lines is an extrapolation of $G^{\prime}$ and $G^{\prime \prime}$ such that the first crossover can be estimated. (b) Evolution of $G_{\max }^{\prime}$ with concentration. Solid and dashed lines represent the tube model predicted evolution of $G_{N}^{0}$ with $\phi$ using $\alpha=1$ and $\alpha=1.3$, respectively. (c) Evolution of $\tau_{d}$ with concentration. Solid and dashed lines represent scaling laws of $\tau_{d}$ predicted by the tube model using $\alpha=1$ and $\alpha=1.3$, respectively. 


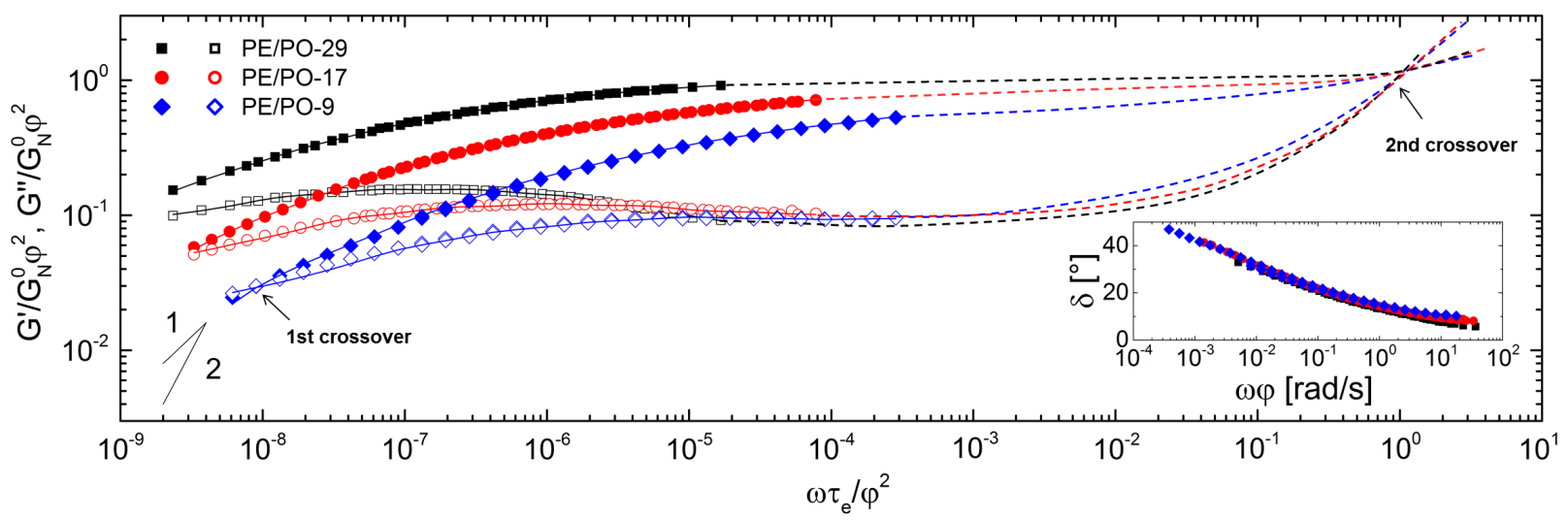

Figure 4: Nondimensional linear response of PE/PO solutions. Closed symbols represent normalized storage modulus and open symbols represent normalized loss modulus. Solid lines represent best fit of the multi mode Maxwell model and dashed lines are guides to the eye to illustate the overlap of the second crossover by normalization and thus the extent of the plateau. The insert shows the phase angle versus frequency shifted by concentration.

$G_{N}^{0} \phi^{1+\alpha}$ assuming $\alpha=1$. Horizontally, the data is normalized by the relaxation time of one entanglement $\tau_{e}$ which can be estimated experimentally from the second crossover. As already mentioned, the limited range of accessible frequencies using SAOS, does not allow for the second crossover to be determined experimentally. Instead, we approximate $\tau_{e}$ using previously given scaling laws of dilution derived from the tube model combined with the value $\tau_{e}=1.1 \cdot 10^{-8} \mathrm{~s}$ for undiluted $\mathrm{PE}$.

The data in Figure 4 have been normalized such that the hypothetical second crossover for the three samples overlap at $\omega \tau_{e} / \phi^{2}=1$. Fixing the second crossover of all samples at the same position reveals that the plateau region (the frequency range between the first crossover and the second estimated crossover at $\omega \tau_{e} / \phi^{2}=1$ ), most likely, stretches over 8 to 10 decades. The large span of the plateau confirms that the samples are highly entangled. The sample of highest concentration (PE/PO-29) shows the widest span of the plateau region, indicating the highest number of entanglements, while the sample of lowest concentration shows the narrowest plateau region (PE/PO-9) indicating the lowest number of entanglements. This trend is in accordance with values given in Table 1. Another way to normalize frequency is by the reptation time $\tau_{d} \propto \phi .{ }^{15,18}$ The insert in Figure 4 shows the phase angle $\delta$ versus $\omega \phi$. 
Curves for all three samples overlap at low frequencies suggesting that the solutions are well mixed and homogeneous in the linear regime.

\section{Uniaxial Extensional Behavior}

The extensional stress growth coefficient $\eta_{E}^{+}$for samples stretched at $150{ }^{\circ} \mathrm{C}$ and $170^{\circ} \mathrm{C}$ is shown in Figure 5a and b, respectively. The extensional response at the two temperatures differs significantly. While most samples at $150^{\circ} \mathrm{C}$ show an undershoot followed by strain hardening, most samples at $170^{\circ} \mathrm{C}$ simply strain soften.

The observed behavior in Figures 5a and $\mathrm{b}$ is unusual for several reasons. First, the qualitative behavior at the two temperatures is not the same throughout the entire stretch. It means that time temperature superposition principle (TTS) does not apply, which it normally does for homogeneous polymeric liquids. ${ }^{19,20}$ Second, while strain softening and stress undershoot is a standard behavior for polymers in shear, it is unusual in extension. ${ }^{21}$ Third, none of the stretch experiments reach a steady state extensional viscosity. ${ }^{22,23}$ The three identified abnormalities (TTS failure, strain softening and absent steady state) suggests that dynamics other than standard chain dynamics are present. As already mentioned PE/PO systems are known to phase separate upon deformation in shear. Under certain conditions this phase separation induces crystallization as well. The high-speed imaging of the filaments during stretching suggests that in extension both phase separation and crystallization occur and, indeed, cause the observed unusual behavior. Overall we define two fundamentally different scenarios encountered during stretching. Scenario 1: Phase separation and Scenario 2: Flow induced crystallization. All samples undergo either Scenario 1 or 2 during stretching depending on the imposed conditions. Following is a detailed description of the two scenarios.

Scenario 1 - Phase Separation Samples stretched at $170^{\circ} \mathrm{C}$ appear to phase separate at $\varepsilon>0.9$ causing the observed strain softening of the samples Figure 5b. Cromer et al. have 

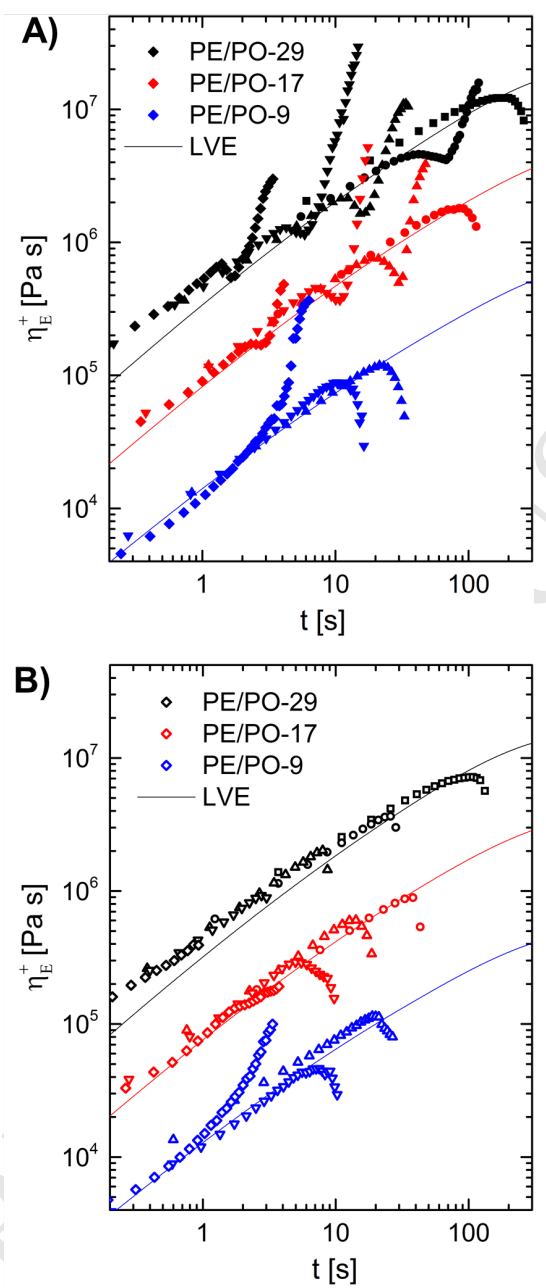

Figure 5: Nonlinear extensional response of PE/PO-solutions at constant Hencky strain rates. (a) Response at $150{ }^{\circ} \mathrm{C}$. Hencky strain rates (from left to right). PE/PO29 (Black symbols): $\dot{\varepsilon}=1,0.3,0.1,0.03,0.01 \mathrm{~s}^{-1}, \mathrm{PE} / \mathrm{PO}-17$ (Red symbols): $\dot{\varepsilon}=$ $1,0.3,0.1,0.03 \mathrm{~s}^{-1}, \mathrm{PE} / \mathrm{PO}-9$ (Blue symbols): $\dot{\varepsilon}=1,0.3,0.1 \mathrm{~s}^{-1}$. (b) Response at $170^{\circ} \mathrm{C}$. Hencky strain rates (from left to right). PE/PO-29 (Black symbols): $\dot{\varepsilon}=$ $1.4,0.41,0.14,0.041,0.014 \mathrm{~s}^{-1}, \mathrm{PE} / \mathrm{PO}-17$ (Red symbols): $\dot{\varepsilon}=1.5,0.44,0.15,0.044 \mathrm{~s}^{-1}$, PE/PO-9 (Blue symbols): $\dot{\varepsilon}=1.5,0.44,0.15 \mathrm{~s}^{-1}$. Lines represent the linear viscoelastic envelope obtained from the multimode Maxwell model. 
investigated conditions for amplification of concentration fluctuations in polymer solutions undergoing planar extensional flow. ${ }^{24}$ Using a two-fluid model with a Rolie-Poly model for the polymer, they predict growth of concentration fluctuations in steady extensional flow with amplification peaks near $\dot{\epsilon} \sim \tau_{d}$ and $\dot{\epsilon} \sim \tau_{R}$ where $\tau_{d}$ and $\tau_{R}$ are the reptation time and the Rouse time for the Rolie-Poly model, respectively. In our experiments we have a broad spectrum of relaxation times with stretch rates within the corresponding range. It is therefore plausible that the same mechanism is responsible for growth of concentration fluctuations in our experiments once a significant orientation of the polymer chains has been established, which is to be expected around a Hencky strain of unity in agreement with our observations for onset of turbidity. We propose that the phase separation and eventual filament disintegration is the ultimate result of the concentration fluctuation.

The phase separation and thus the course of deformation differs depending on the imposed rate and the concentration of the sample. For samples stretched at $170^{\circ} \mathrm{C}$, the largest difference in behavior is observed between samples of high concentration stretched at low rates (Figure 6a) and samples of low concentration stretched at high rates (Figure 6b).

For samples of high concentration, stretched at low rates, the phase separation induces multiple large cracks in the midfilament region (see Figure 6a), a behavior reminiscent of crazing. Filament failure follows immediately after, disabling Hencky strains $\varepsilon>1.5$ to be reached. As such, the rapid filament failure cannot be considered a brittle fracture. ${ }^{25}$ The reason being that the failure is not caused by a single crack propagation, but by multiple cracks propagating simultaneously.

Samples of low concentration, stretched at high rates phase separate as well (see Figure 6b). As opposed to the above mentioned case, the range of accessible strains as well as the type of filament failure differs.

Phase separation in Figure 6b is identified as a slight onset of turbidity in the midfilament region at $\varepsilon \approx 0.9$. The phase separation propagates at increasing $\varepsilon$ seen as an increase in turbidity and at $\varepsilon>3$ the phase separation is so pronounced that the surface of the filament 


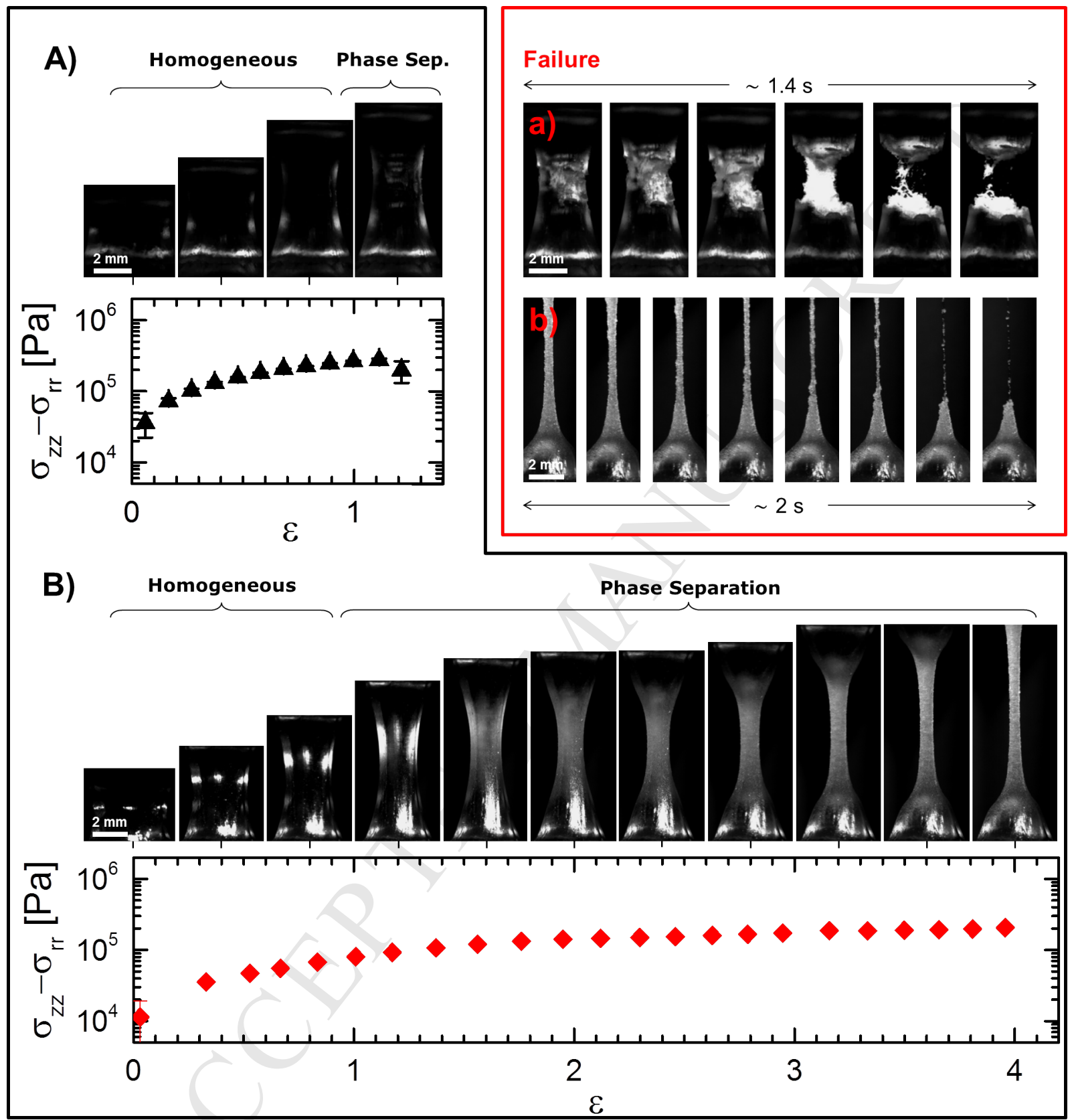

Figure 6: Examples of rheological response and corresponding images (black frame) as well as failure (red frame) of $\mathrm{PE} / \mathrm{PO}$ filaments elongated at $170^{\circ} \mathrm{C}$. (a) For a sample of high concentration stretched at low rates. That is PE/PO-29 stretched at $\dot{\varepsilon}=0.014 \mathrm{~s}^{-1}$. (b) For a sample of lower concentration stretched at high rates. That is $\mathrm{PE} / \mathrm{PO}-17$ stretched at $\dot{\varepsilon}=1.5 \mathrm{~s}^{-1}$. The bright spots near the edges are due to light source reflections from the filament surface. 
becomes uneven and lumpy. Failure of the filament occurs through thinning of the filament during which, phase separation is even more evident.
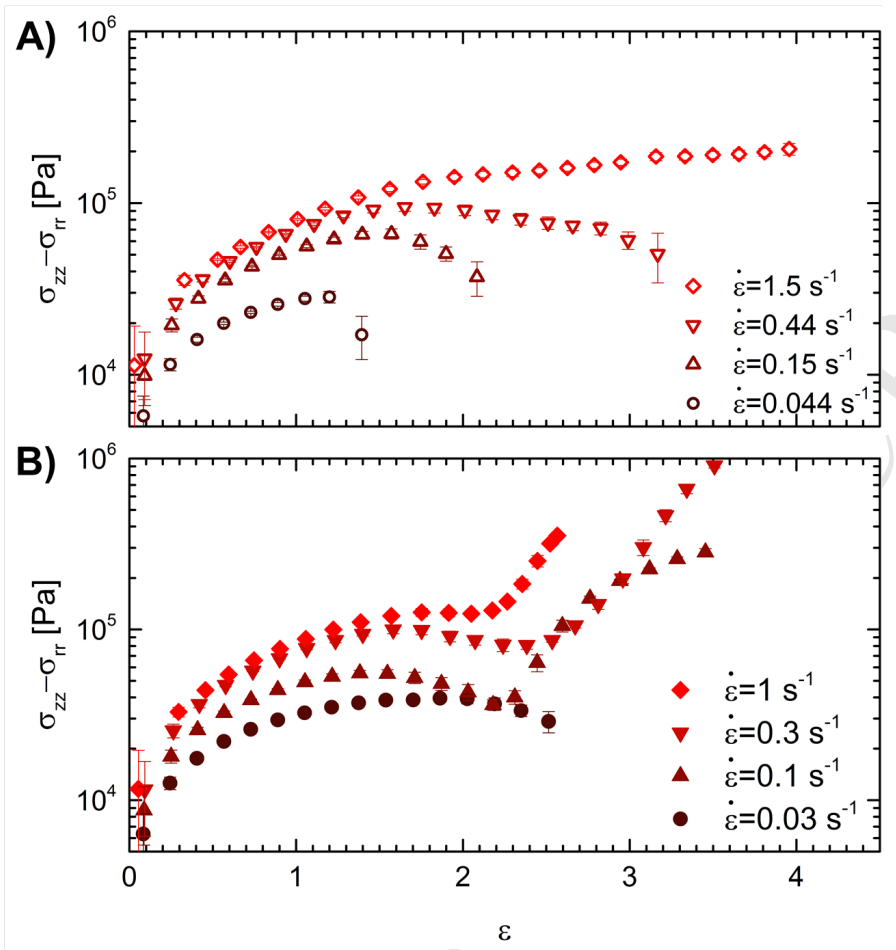

Figure 7: Extensional stress versus Hencky strain for stretches of PE/PO-17 at a) $170^{\circ} \mathrm{C}$ and b) $150^{\circ} \mathrm{C}$.

The stretch experiments presented in Figures 6a and 6b show the extremes of Scenario 1. All samples stretched at $170{ }^{\circ} \mathrm{C}$ show a behavior somewhere in between the two presented extremes. Figure 7a shows the gradual transition from one extreme to the other for PE/PO17 and the following two trends should be noted. 1) With increasing $\dot{\varepsilon}$ the degree of strain softening decreases. 2) With increasing $\dot{\varepsilon}$ the maximum $\varepsilon$ that can be reached before failure increases. We hypothesize that both trends are a result of an increased chain stretch for increasing $\dot{\varepsilon}$ stabilizing the filament as opposed to the observed phase separation.

Scenario 2 - Flow Induced Crystallization The strain hardening behavior of solutions stretched at $150{ }^{\circ} \mathrm{C}$, originates from FIC. No samples stretched at $170^{\circ} \mathrm{C}$ show signs of FIC. 


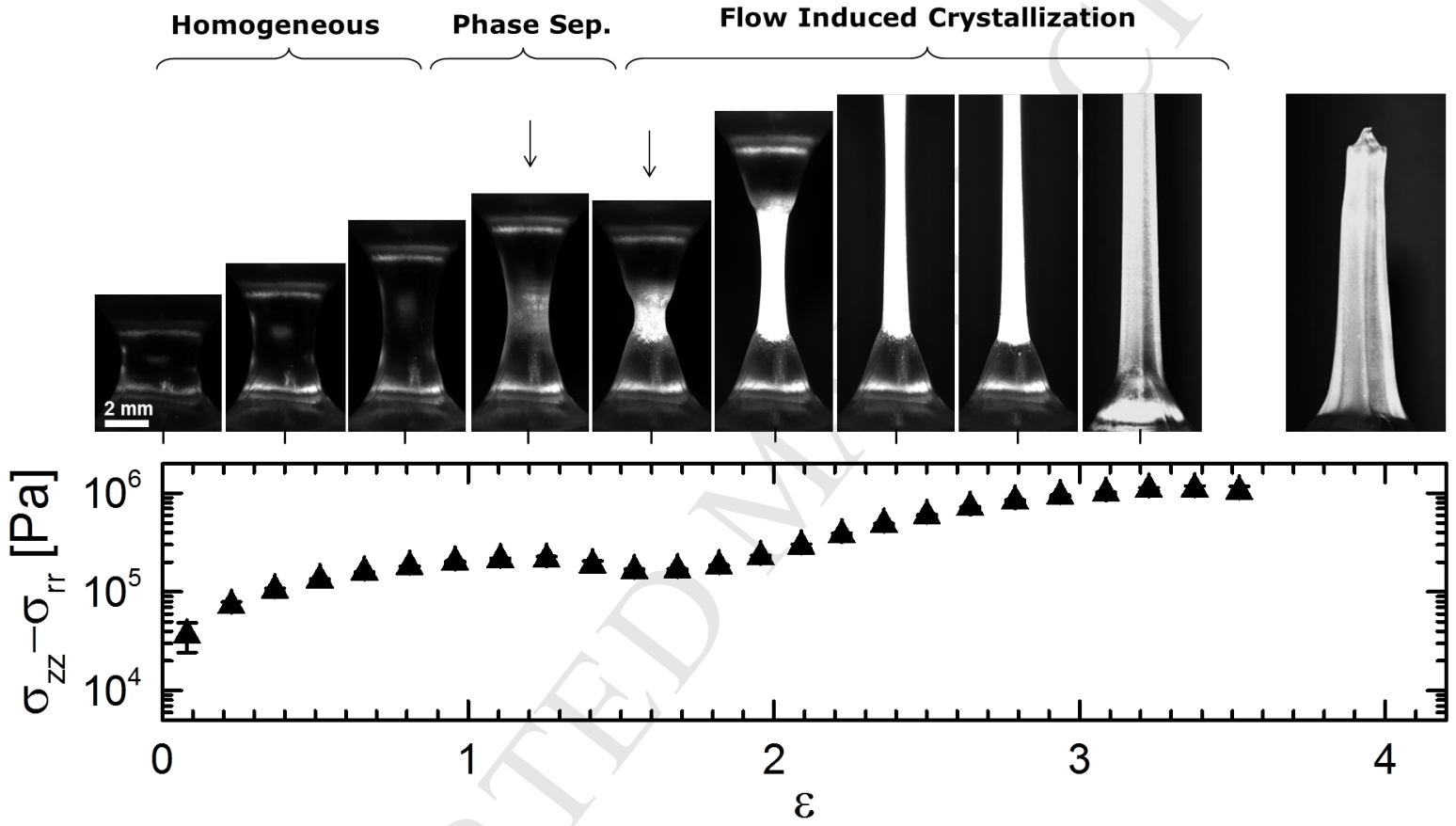

Figure 8: Example of FIC for stretch experiments performed at $150{ }^{\circ} \mathrm{C}$. The sample is $\mathrm{PE} / \mathrm{PO}-29$ stretched at $\dot{\varepsilon}=0.1 \mathrm{~s}^{-1}$. Images are collected through crossed polarizers with light source and camera on the same side. Arrows above image 4 and 5 indicate the strain range in which the onset of FIC occurs. The two images to the far left are taken from a replica of the stretch. 
An example of a stretch in which FIC occurs, is shown in Figure 8. Analogous to Scenario 1 , the sample is still believed to undergo phase separation at $\varepsilon \approx 0.9$, due to the observed strain softening behavior combined with the onset of turbidity. At $\varepsilon \approx 1.5$ the midfilament region of the sample transforms from turbid to opaque while the stress starts to rise again. Both observations suggest onset of flow induced crystallization (FIC). An additional indication of FIC is the spontaneous midfilament decrease observed between frames 4 and 5 (indicated by arrows) in Figure 8. For a fast spontaneous diameter decrease the highly sensitive control scheme will counter act the apparent deformation by pushing downward which is indeed the case as observed by the non-monotone motion between frames 4, 5 and 6. Flow induced crystallization can cause such a spontaneous decrease due to the density increase upon crystallization. While this change might seem negligible, it is not. A density increase of $\approx 20 \%$ between molten and semicrystalline HDPE would yield a $\approx 7 \%$ increase in density for the $29 \mathrm{w} \%$ solution of PE in PO. This corresponds to an apparent Hencky strain of 0.06 . Given that the decrease in Figure 8 occurs on the time scale of $\sim 2 \mathrm{~s}$, the FIC contribution to the Hencky strain rate is $0.03 \mathrm{~s}^{-1}$. Relative to the imposed Hencky strain rate of $0.1 \mathrm{~s}^{-1}$, this a significant contribution that does affect the movement of the top plate. Combining all three observations (turbid-opaque transition, density increase and strain hardening) it is very likely that flow induced crystallization takes place. At $\varepsilon>1.5$ a crystallized neck region develops from the midfilament region causing the significant strain hardening behavior. The transition from molten sample near the plates to the neck area is characterized by an abrupt decrease in diameter along side a sharp transition from transparent to opaque. While the midfilament is deformed the neck area is extended axially by pulling from the reserve of molten sample at the end plates and into the crystallizing neck. Hence the reserve of molten sample gradually decreases. At $\varepsilon>3$ most of the sample is incorporated into a long crystallized neck. At this point the control scheme starts to fail, not because of an uneven filament, but because the speed of the top plate reaches its maximum speed. Furthermore end plate instability is observed due to the high deformation rates close 
to the plates. ${ }^{26}$ At sufficiently high stresses the filament fractures in a brittle manner. DSC measurements of the the midfilament region after stretch for a sample stretched under same conditions as in Figure 8 showed a rise in $T_{m}$ of $2.8^{\circ} \mathrm{C}$. The increase in $T_{m}$ is further proof that FIC has taken place. ${ }^{27}$

Figure $7 \mathrm{~b}$ shows the stress response of $\mathrm{PE} / \mathrm{PO}-17$ at various $\dot{\varepsilon}$. The stress undershoot is less pronounced for increasing $\dot{\varepsilon}$ suggesting a higher degree of chain stretch, similar to Scenario 1. Samples stretched $150^{\circ} \mathrm{C}$ at the lowest rates (i.e. experiments that do not show strain hardening) seem to follow Scenario 1, as well.

\section{Significance of Time Temperature Superposition Principle}

The high-speed imaging reveals that at small deformations $\varepsilon<0.9$ all samples appear transparent both in Scenario 1 and Scenario 2. The transparency suggests that the samples are homogeneous (i.e. no phase separation or FIC) at strains $\varepsilon<0.9$. To confirm homogeneity, we compare the normalized extensional response measured at $150{ }^{\circ} \mathrm{C}$ and $170^{\circ} \mathrm{C}$ (see Figures $9 \mathrm{a}, \mathrm{b}$ and $\mathrm{c})$. Here we assume that TTS works also for the nonlinear response. The characteristic time constant used in the normalization is the average Rouse relaxation time $\tau_{R}=\tau_{e} Z^{2}$. As $\tau_{e} \propto \phi^{-2}$ and $Z^{2} \propto \phi^{2}$ it is evident that $\tau_{R}$ is independent of dilution and thus all samples have the same value of $\tau_{R}$. We use previously defined values of the undiluted UHMwPE to estimate $\tau_{R}$ for the solutions. At $150{ }^{\circ} \mathrm{C}$ we obtain $\tau_{R}=0.11 \mathrm{~s}$ and with a shift factor $a_{T}=1.47$, we get $\tau_{R}=0.075 \mathrm{~s}$ at $170^{\circ} \mathrm{C}$. The normalized stretch rates are given in terms of the Rouse Weissenberg number $W i_{R}=\dot{\varepsilon} \tau_{R}$. It weighs the rate of deformation relative to the rate of relaxation of the weight average polymer chain of the sample. Keeping in mind that the sample is highly polydisperse and that $\tau_{R}$ is based on the weight average molar mass, the fractions of higher molar mass in the samples will have much higher $\tau_{R}$. It is thus possible to observe nonlinear behavior even at $W i<1$.

At small strains, the normalized extensional behavior of all samples in Figures 9a, b and c follow the LVE and obey TTS. The fact that the normalized extensional response follows 


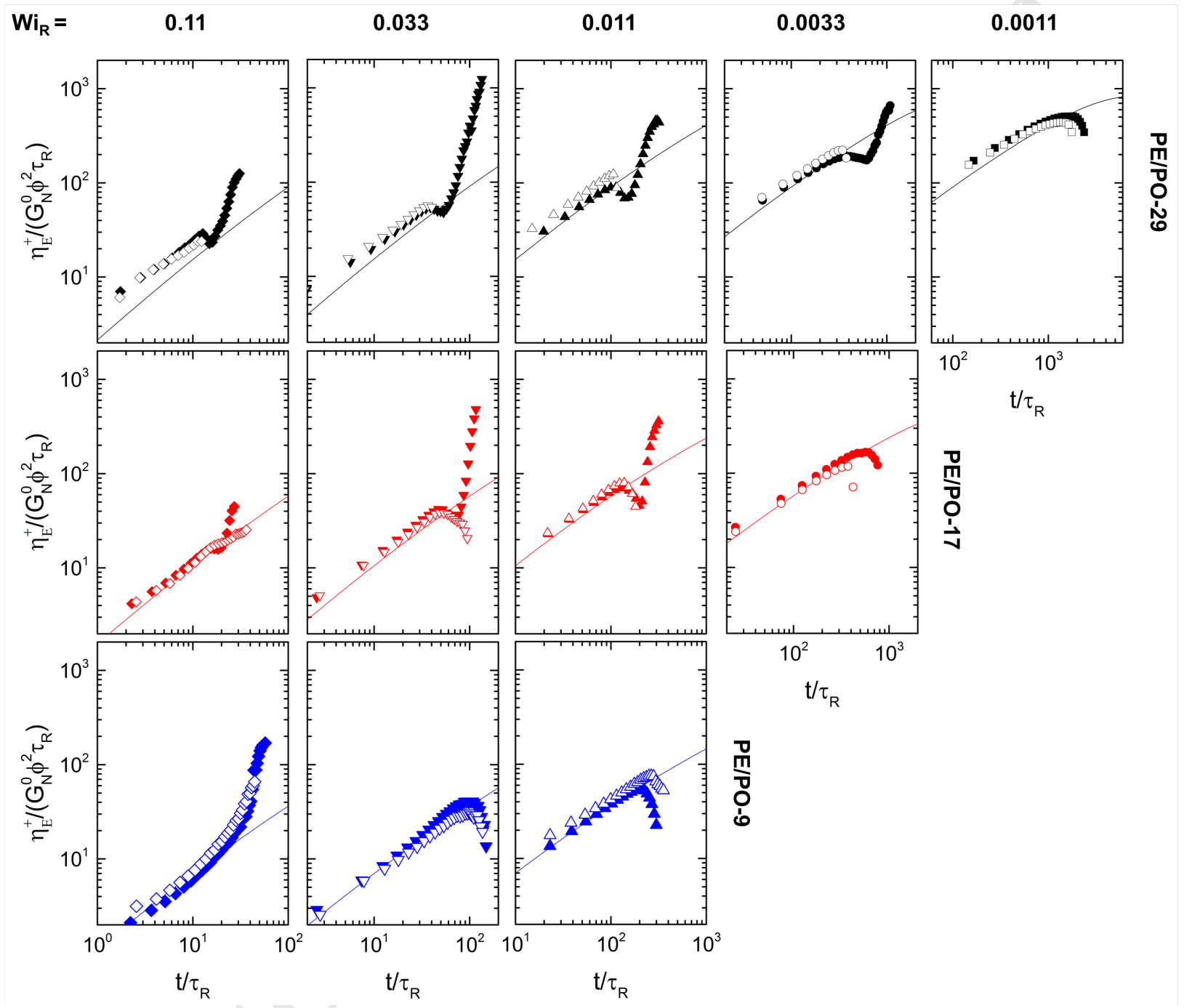

Figure 9: Normalized nonlinear extensional response at various Weissenberg numbers for $\mathrm{PE} / \mathrm{PO}-29$ (top row), PE/PO-17 (middle row) and PE/PO-9 (bottom row). Closed symbols indicate stretches performed at $150^{\circ} \mathrm{C}$. Open symbols indicate stretches performed at $170^{\circ} \mathrm{C}$. Weissenbeg numbers apply to both open and closed symbols. 
the LVE and obey TTS confirms that at small deformations the samples are homogeneous and that the rheology is governed solely by chain dynamics. We observe that in many cases TTS even holds in the strain softening region. This suggests that although the samples are phase separated, the response is still governed by chain dynamics only. However at larger strains the breakdown of TTS confirms that an additional phenomenon (in this case FIC) with a temperature dependency different from that of chain dynamics, occurs. The onset of FIC move towards earlier times for increasing $W i_{R}$. This is in accordance with general theories stating that the onset of FIC is governed by chain stretch. ${ }^{28}$

\section{Conclusion}

Solutions of UHMwPE in extensional flow show a rich variety of behavior at temperatures well above $T_{m}$. We find that at $\varepsilon>0.9$ the extensional behavior differs fundamentally with concentration, temperature and imposed deformation rate. Overall the observed behaviors can be divided in two Scenarios 1 - Phase separation and 2 - FIC. At $150^{\circ} \mathrm{C}$ flow induced crystallization (Scenario 2) was observed for high $W i_{R}$ and high $\phi$. The higher the concentration, the lower the $W i_{R}$ at which FIC can be detected. E.g. for PE/PO-29, FIC is observed at $W i_{R}$ as low as 0.0033 , while for $\mathrm{PE} / \mathrm{PO}-9$, the lowest $W i_{R}$ at which FIC is observed is 0.11 . The time at which onset of FIC is observed, decrease for increasing $W i_{R}$. The earlier onset of FIC upon increasing $W i_{R}$ is in accordance with the general belief that FIC is governed by chain stretch that increases with $W i_{R}$ as well. At lower $W i_{R}$ and $\phi$ the filament only appeared to phase separate (Scenario 1). At $170^{\circ} \mathrm{C}$ all samples followed Scenario 1. The observed flow induced phenomena (phase separation and crystallization) are in general agreement with previous studies performed in controlled shear and along a fiber spin-line in which, however, the deformation cannot be controlled. To reach a better understanding controlled extensional rheology in combination with in-situ light scattering or even X-ray scattering will be needed. 


\section{Acknowledgement}

The authors thank Aage og Johanne Louis-Hansen fonden for financial support as well as Prof. Dimitris Vlassopoulos for fruitful discussions on the work.

\section{Supporting Information Available}

The following files are available free of charge.

- SupportingInformation.pdf: Supporting figures S1 and S2

\section{References}

(1) Keller, A.; Kolnaar, H. W. H. Materials science and technology; VCH Verlagsgesellschaft mbH, 1997; pp $187-268$.

(2) Hsiao, B. S.; Yang, L.; Somani, R. H.; Avila-Orta, C. A.; Zhu, L. Unexpected ShishKebab Structure in a Sheared Polyethylene Melt. Phys. Rev. Lett. 2005, 94, 117802.

(3) Ohta, Y.; Murase, H.; Sugiyama, H.; Yasuda, H. Non-newtonian rheological behavior of semi-dilute ultra-high molecular weight polyethylene solution in gel-spinning process. 1: Concentration effect on the fundamental rheological properties. Polymer Engineering Es Science 2000, 40, 2414-2422.

(4) Murase, H.; Kume, T.; Hashimoto, T.; Ohta, Y.; Mizukami, T. Shear-Induced Concentration Fluctuations in Ultrahigh Molecular Weight Polyethylene Solutions. 1. Observation above the Melting Point. Macromolecules 1995, 28, 7724-7729.

(5) Murase, H.; Kume, T.; Hashimoto, T.; Ohta, Y. Shear-Induced Structures in Semidilute Solution of Ultrahigh Molecular Weight Polyethylene at Temperature Close to Equilibrium Dissolution Temperature âĂă. Macromolecules 2005, 38, 6656-6665. 
(6) Murase, H.; Kume, T.; Hashimoto, T.; Ohta, Y. Time Evolution of Structures under Shear-Induced Phase Separation and Crystallization in Semidilute Solution of Ultrahigh Molecular Weight Polyethylene âĂă. Macromolecules 2005, 38, 8719-8728.

(7) Murase, H.; Ohta, Y.; Hashimoto, T. A New Scenario of Shish-Kebab Formation from Homogeneous Solutions of Entangled Polymers: Visualization of Structure Evolution along the Fiber Spinning Line. Macromolecules 2011, 44, 7335-7350.

(8) Pennings, A. J.; van der Hooft, R. J.; Postema, A. R.; Hoogsteen, W.; ten Brinke, G. High-speed gel-spinning of ultra-high molecular weight polyethylene. Polymer Bulletin 1986, 16, 167-174.

(9) Pennings, A. Further studies on the high-speed gel-spinning of ultra-high molecular weight polyethylene. Polymer Bulletin 1990, 23.

(10) Das, C.; Inkson, N. J.; Read, D. J.; Kelmanson, M. a.; McLeish, T. C. B. Computational linear rheology of general branch-on-branch polymers. Journal of Rheology 2006, 50, 207.

(11) Flory, P. Principles of Polymer Chemistry; Cornell University Press,, 1953.

(12) Román Marín, J. M.; Huusom, J. K.; Alvarez, N. J.; Huang, Q.; Rasmussen, H. K.; Bach, A.; Skov, A. L.; Hassager, O. A control scheme for filament stretching rheometers with application to polymer melts. Journal of Non-Newtonian Fluid Mechanics 2013, $194,14$.

(13) Bach, A.; Almdal, K.; Rasmussen, H. K.; Hassager, O. Elongational Viscosity of Narrow Molar Mass Distribution Polystyrene. Macromolecules 2003, 36, 5174.

(14) Rasmussen, H. K.; Bejenariu, A. G.; Hassager, O.; Auhl, D. Experimental evaluation of the pure configurational stress assumption in the flow dynamics of entangled polymer melts. J. Rheol. 2010, 54, 1325. 
(15) Huang, Q.; Mednova, O.; Rasmussen, H. K.; Alvarez, N. J.; Skov, A. L.; Almdal, K.; Hassager, O. Concentrated Polymer Solutions are Different from Melts: Role of Entanglement Molecular Weight. Macromolecules 2013, 46, 5026-5035.

(16) van Ruymbeke, E.; Masubuchi, Y.; Watanabe, H. Effective Value of the Dynamic Dilution Exponent in Bidisperse Linear Polymers: From 1 to 4/3. Macromolecules 2012, 45, 2085-2098.

(17) Fetters, L. J.; Lohse, D. J.; Colby, R. H. In Physical Properties of Polymers, 2nd ed.; 2, Ed.; Springer: New York, 2007; Chapter 25, p 1073.

(18) Doi, M. Introduction to polymer physics; Clarendon Press, 1996; p 120.

(19) Dealy, J.; Plazek, D. Time-temperature superposition - a users guide. Rheol. Bull. 2009, $78,16$.

(20) Kapnistos, M.; Hinrichs, A.; Vlassopoulos, D.; Anastasiadis, S. H.; Stammer, A.; Wolf, B. A. Rheology of a Lower Critical Solution Temperature Binary Polymer Blend in the Homogeneous, Phase-Separated, and Transitional Regimes. Macromolecules 1996, 29, 7155-7163.

(21) Costanzo, S.; Huang, Q.; Ianniruberto, G.; Marrucci, G.; Hassager, O.; Vlassopoulos, D. Shear and Extensional Rheology of Polystyrene Melts and Solutions with the Same Number of Entanglements. Macromolecules 2016, 49, 3925-3935.

(22) Huang, Q.; Hengeller, L.; Alvarez, N. J.; Hassager, O. Bridging the Gap between Polymer Melts and Solutions in Extensional Rheology. Macromolecules 2015, 48, 41584163.

(23) Huang, Q.; Alvarez, N. J.; Matsumiya, Y.; Rasmussen, H. K.; Watanabe, H.; Hassager, O. Extensional Rheology of Entangled Polystyrene Solutions Suggests Importance of Nematic Interactions. ACS Macro Letters 2013, 2, 741. 
(24) Cromer, M.; Villet, M. C.; Fredrickson, G. H.; Gary Leal, L.; Stepanyan, R.; Bulters, M. J. H. Concentration fluctuations in polymer solutions under extensional flow. Journal of Rheology 2013, 57, 1211-1235.

(25) Huang, Q.; Alvarez, N. J.; Shabbir, A.; Hassager, O. Multiple Cracks Propagate Simultaneously in Polymer Liquids in Tension. Phys. Rev. Lett. 2016, 117, 87801.

(26) Bach, A.; Rasmussen, H. K.; Longin, P.-Y.; Hassager, O. Growth of non-axisymmetric disturbances of the free surface in the filament stretching rheometer: experiments and simulation. Journal of Non-Newtonian Fluid Mechanics 2002, 108, 163.

(27) Hsiao, B. S.; Zuo, F.; Mao, Y.; Schick, C. Handbook of Polymer Crystallization; John Wiley \& Sons, Inc., 2013; pp 1-30.

(28) Roozemond, P. C.; Peters, G. W. M. Flow-enhanced nucleation of poly(1-butene): Model application to short-term and continuous shear and extensional flow. Journal of Rheology 2013, 57, 1633-1653. 


\section{Graphical TOC Entry}

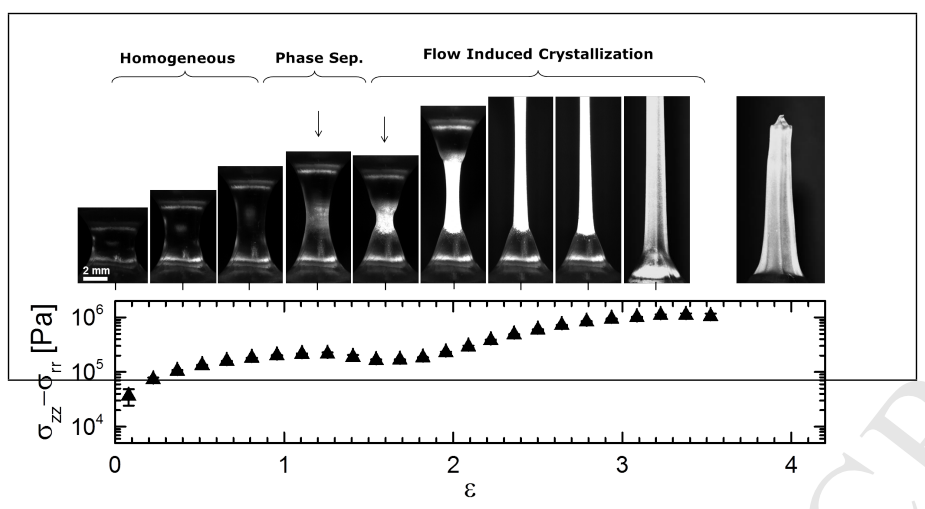




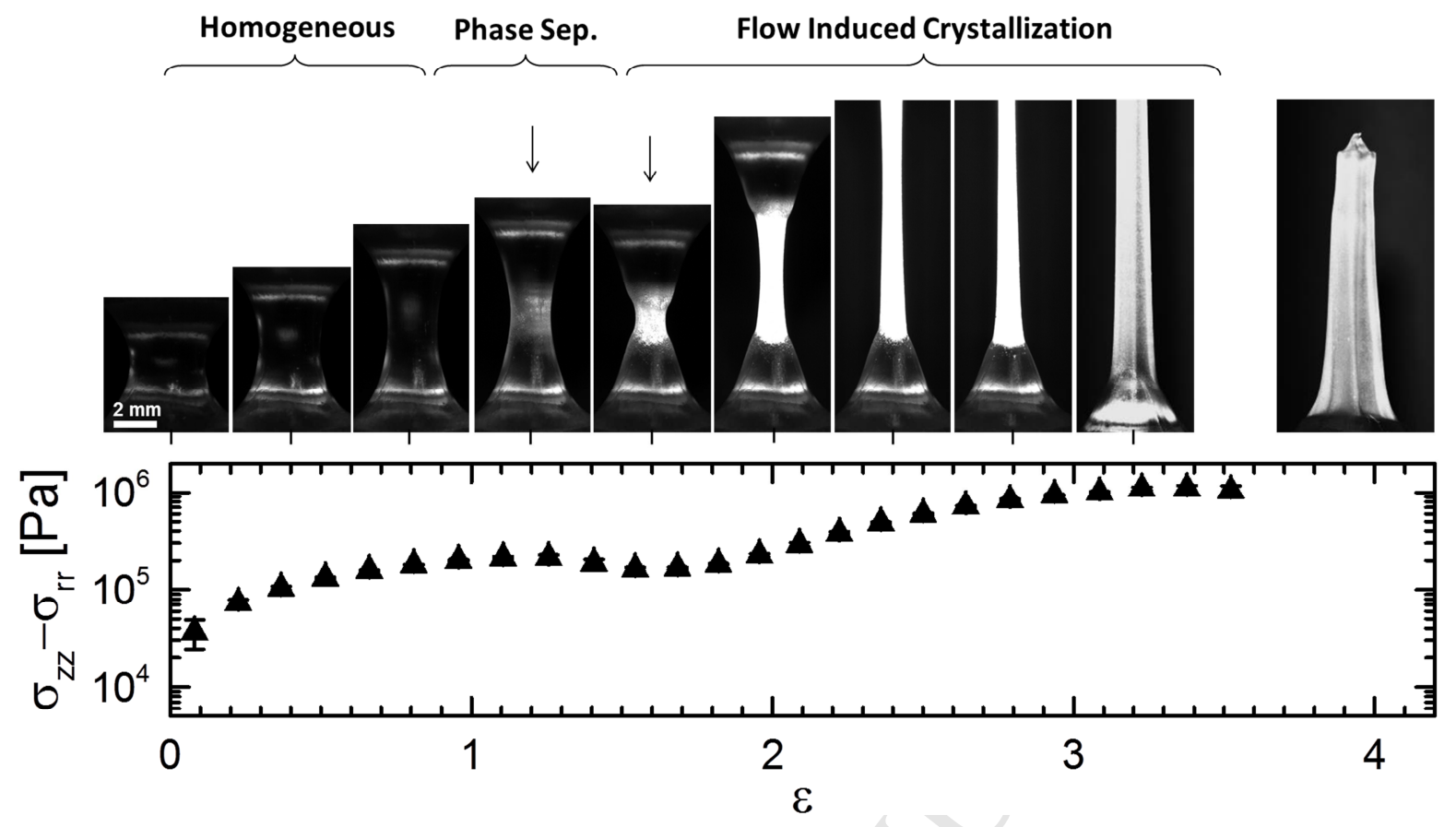




\section{ACCEPTED MANUSCRIPT}

Extension Induced Phase Separation and Crystallization in Semidilute Solutions of Ultra High Molecular Weight Polyethylene

by

Sara Lindeblad Wingstrand,y Luna Imperiali,z Roman Stepanyan,z and Ole Hassager

\section{Highlights}

- Solutions of Ultra High Molecular Weight Polyethylene (UHMwPE) in paraffin oil (PO) shows crystallization at temperatures well above the crystalline melting point when subjected to strong extensional flow.

- Two phenomena have been observed during extension

- Scenario 1: Flow induced phase separation. This is strongly destabilizing.

- Scenario 2: Flow induced crystallization. This is strongly stabilizing. 